\title{
A microcomputer-based pause analysis apparatus
}

\author{
STEVEN T. GOODING \\ Xerox Corporation, Webster, New York 14580
}

and

\section{THOMAS GOODING and J. NATHAN SWIFT \\ State University of New York, Oswego, New York 13126}

\begin{abstract}
The study of pauses in human speech is an important component of the psycholinguistic analysis of speech patterns. Since the inception of this type of research, problems in instrumentation have presented a number of concerns. These problems have been well documented in the literature in pausology. While accurate instrumentation has been developed by several research teams, cost of such equipment has been high, and hand recording of data has been required. This report describes a microcomputer-based pause analysis apparatus, which enables the measurement of pause times precise to better than $.01 \mathrm{sec}$ at less cost than previous tochnology. The apparatus consists of analog hardware and digital computer hardware and software. A printout provides a permanent record of pause times keyed on a line-by-line basis to transcripts. Flexibility is built in by means of a BASIC program enabling specifications for selections of pauses between words, phrases, or sentences based on pause duration.
\end{abstract}

The study of pauses in human speech is an important aspect of the psycholinguistic analysis of speech patterns. Some researchers are interested in the pauses that divide words and phrases; others are interested in wait time, the pauses that occur between questions and responses. Recent research on pauses in interactive speech in educational settings has made accurate measurement of pauses in dialogue imperative. However, instrumentation in the field of pausology has presented serious problems to researchers. As early as 1965 , Goldman-Eisler called attention to the fact that rectifier devices available in those early stages of pausology research were inadequate for reliable monitoring of speech pauses. Shortly thereafter, improvements in instrumentation utilizing a decade counter (Hewlett-Packard AC-4A/B) and a digital recorder (Hewlett-Packard 560A) were reported (Goldman-Eisler, 1968). In subsequent years, pause measurement instrumentation has continued to improve.

\section{PROBLEM}

Because of instrumentation costs, pausology research has made widespread use of subjective judgment in monitoring the location and frequency of unfilled pauses, even in more recent studies (Braehler \& Zenz, 1975; O'Connell \& Kowal, 1981). These authors have

This research was supported in part by National Science Foundation Grant SED 8015819, J. Nathan Swift, principal investigator. Findings and opinions expressed in this paper are solely the responsibility of the authors. Information concerning sof tware, including program listings, is available from the authors. noted that more reliable and valid measures, such as those produced by an audio-frequency spectrometer and level recorder, are imperative if progress is to be made. O'Connell and Kowal have found that such instrumentation as the Briel and Kjaer audio-frequency spectrometer (Type 2112) and level recorder (Type 2305) provides a precise and objective measure of pauses. Problems remain, however, because even sophisticated apparatus requires laborious and time-consuming hand recording of data, and equipment costs are high.

The newly developed apparatus described in this report represents an advance in pause measurement instrumentation, since it provides an accurate measure of pause time in speech and does so at considerably less cost than earlier technology did. This apparatus was designed and constructed as part of a National Science Foundation funded research project in science education. The project, which deals with teacher pauses in questioning behavior and subsequent effects on student behavior, further extends and evaluates the pioneering work of Rowe (1974) on pause patterns of teachers. The study, consisting of audio-tape data from 40 science classes, produced $6,000 \mathrm{~min}$ of audio-taped transcriptions. Faced with such a large amount of data for pause-time analysis, it was imperative that an automated pause analysis system be developed.

\section{INSTRUMENTATION}

The complete pause measurement package incorporates analog hardware and digital computer hardware and software. The analog components are composed of 
a solenoid-controlled audio-cassette tape deck (NAD Model 6140), a 10-band graphic frequency equalizer (Radio Shack Model 31-2000), an audio-signal restoration unit (Symmetric Sound Systems Model ASRU), and a level-crossing detection circuit as diagrammed in Figure 1. The digital hardware features a microcomputer that combines a 6502 microprocessor, an LED display, a thermal printer (Rockwell AIM 65 microcomputer), and a custom digital interface circuit described in Figure 1. The computer software is composed of a high-speed assembly language subroutine and a BASIC language program designed to search, measure, record, tabulate, sum, and average pauses in prerecorded speech. The block diagram in Figure 2 shows the relationship among the components of the instrumentation package.

The level-crossing detector performs the analog-todigital interface function. It accepts input from the audio tape conditioned by the frequency equalizer and signal restoration unit. The equalizer is set up as a $250-\mathrm{Hz}$ to $1,000-\mathrm{Hz}$ bandpass filter, and the restoration unit provides $8.5 \mathrm{~dB}$ of signal expansion. The output of the level-crossing detector is digital in nature (being $1 \mathrm{~s}$ or $0 \mathrm{~s}$ ). The 1 to 0 transitions correspond directly to the audio transitions of the set detection level. This detection level is variable, thus providing

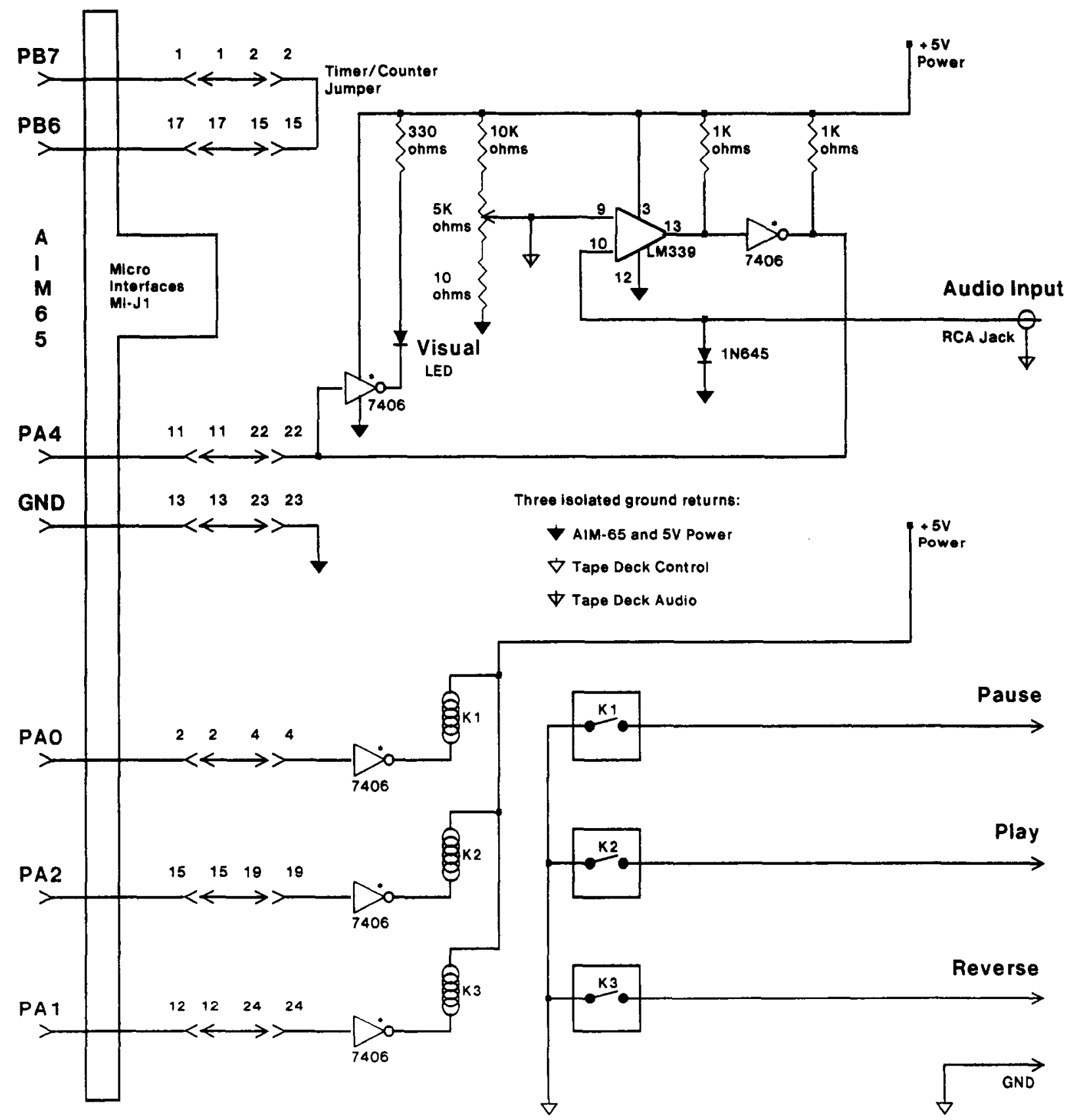

Figure 1. Pause analyzer circuit diagram. 


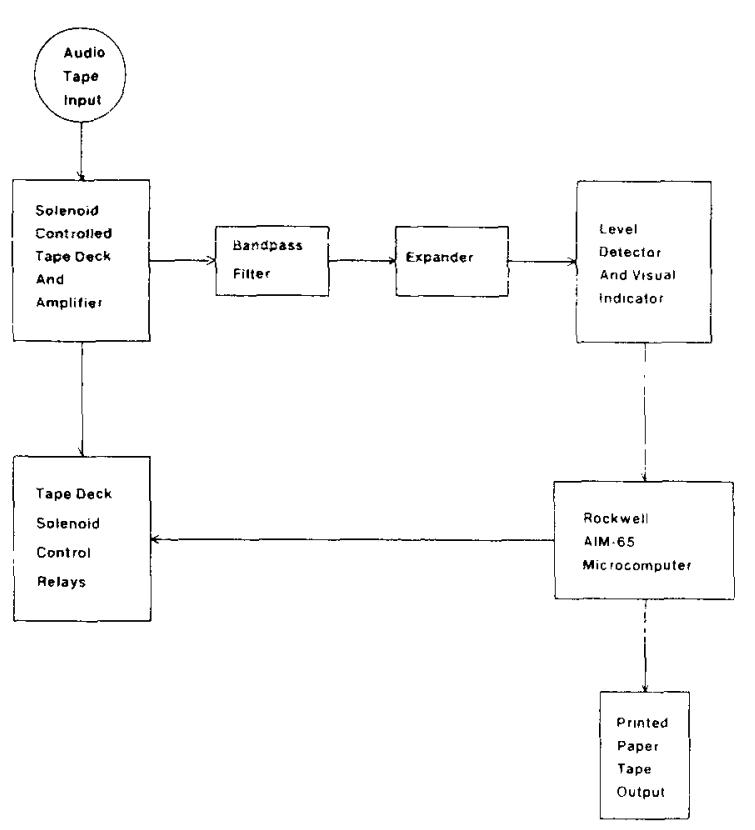

Figure 2. Pause analysis apparatus block diagram.

adjustment to permit tape hiss and background noise rejection.

The output of the level-crossing detector is conditioned by the custom digital interface. Visual indication of level-crossing detection is also provided by this circuit. The output of this interface circuit is fed directly into the AIM 65 microcomputer. The remainder of the custom interface circuit provides computer control of the cassette tape through a simple relay setup.

The assembly language subroutine continuously samples the digital signal from the interface circuit at high speed. Pauses are detected by determining when valid speech stops. Noise bursts during the pause are rejected. When valid speech is again detected, the value representing the total pause time is passed to the BASIC language program. Samples are taken at $.001-\mathrm{sec}$ intervals, and groups of 255 are evaluated. This results in a minimum pause detection time of $.255 \mathrm{sec}$, with $.001 \mathrm{sec}$ detection accuracy after initial pause detection occurs. Thus, all pauses less than $.255 \mathrm{sec}$ are recorded by the assembly language subroutine as $.255 \mathrm{sec}$.
The BASIC program accepts the pause duration values from the assembly language subroutine and converts the value to seconds accurate to three decimal places. The pause duration in seconds and other information is indexed to the speech patterns by operator interaction. This is accomplished through following the dialogue on a transcript as the computer measures the pauses. Valid pauses can then be separated from invalid pauses, noise, or other inappropriate input. Each pause is automatically indexed to the transcript by line number and pause type. Additionally, the cassette tape is started, stopped, and reversed by BASIC subroutines as required. This control is achieved through the simple relay setup in the custom digital interface. Other information and identification can easily be added to the program as required. The paper-tape printout provides a permanent record of the pause times on a line-by-line basis. It also prints the total and mean pause times for each audio tape and accompanying transcript. Thus, the circuit and software in combination perform tape hiss and noise-burst rejection respectively, thereby increasing reliability and validity of the output. Flexibility is built in by means of a parameter that exists in the BASIC program, enabling specifications for selections of pauses between words, phrases, or sentences based on the pause duration. Measurement of pauses is therefore automatic and precise to better than $.01 \mathrm{sec}$. The importance of the $.01-\mathrm{sec}$ precision and other variables involved, such as bandpass, must not be underestimated. In order to compare and support results and findings, care must be taken to preserve these baselines and ensure that a similar metric is used. If this is not the case, then any comparison will be invalid.

\section{REFERENCES}

Braenler, E., \& Zenz, H. Artifacts in the registration and interpretation of speech process variables. Language and Speech, 1975, 18, 166-179.

Goldman-EISLER, F. Psycholinguistics: Experiments in spontaneous speech. London: Academic Press, 1968.

O'Connele, D. C., \& Kowal, S. Pausology. In W. Sedelow and S. Sedlow (Eds.), Computer uses in the study of languages (Vol. 3). Cognitive approaches. The Hague: Mouton, 1981.

Rowe, M. B. Pausing phenomena: Influence on the quality of instruction. Journal of Psycholinguistic Research, 1974, 3 , 203-224. 